Miah, S., Shen, J., Lamp, J., Kerr, D., \& Gammack, J. (2019). Emerging Insights of Health Informatics Research: A Literature Analysis for Outlining New Themes. Australasian Journal of Information Systems, 23. doi:10.3127/ajis.v23i0.2137

\title{
Review
}

This post publication review discusses the systematic review of health informatics by Miah, Shen, Lamp, Kerr, and Gammack (2019), highlighting its contribution to the field and indicating areas for further research.

The reviewed article is a sound contribution to the literature on information systems (IS), adding timely insights into the current state of health informatics guided by a pressing research question: "What are the current research themes in the existing health informatics studies that show promise for future knowledge growth?" Miah et al. (2019) adopted a thematic analysis approach for analysing and classifying the existing literature to summarise new insights that may have future research value. It extends previous health informatics publications in this journal (Burmeister, Islam, Dayhew, \& Crichton, 2015; Carlson, Farrelly, Frazer, \& Borthwick, 2015; Poulsen, 2018; Simpson, Nevile, \& Burmeister, 2003) and other IS journals (Miah, Hasan, \& Gammack, 2017; Miah, Hasan, Hasan, \& Gammack, 2017). Chiefly, the study identified ten research themes regarding existing health informatics by succinctly encapsulating the emerging aspects of this multidisciplinary IS subset.

To comment on the study, we have identified several sub-domains underneath the authors' themes. We have done this to critique and complement the article. That is, we identify topics which are absent in the study, but we use the authors' themes to do so. We hope that this contributes towards achieving the authors' aim to "determine underlying technological developments and their target application areas" (Miah et al., 2019).

Here, we contribute additional topics worthy of further investigation as they fall into the authors' themes. Those are, blockchain (themes: digital health and ethics, privacy, security and other concerns), fog/edge computing (themes: clinical and decision support aspects and digital health), and ethical healthcare robots (themes: telemedicine, telecare and telematics and smart living). Health informatics with blockchain is being done by Kuo, Kim, and Ohno-Machado (2017) and Gordon and Catalini (2018). Health applications of fog/edge computing are being explored by Kraemer, Braten, Tamkittikhun, and Palma (2017) and Barik, Dubey, and Mankodiya (2017). Ethical healthcare robots are being investigated by Vanderelst and Winfield (2018), Poulsen and Burmeister (2019), and Anderson, Anderson, and Berenz (2017).

To briefly expand on just one of these sub-domains and demonstrate the work being done in health informatics themes identified by the authors, consider ethical healthcare robotics. Anderson et al. (2017) developed and empirically tested a value-driven medicine delivery robot which upholds Rossian deontological ethics and uses situational variables to make dynamic ethical decisions. Similarly, Poulsen and Burmeister (2019) are designing ethical care robots for elders which are dynamically responsive to care values as they emerge from human rights, professional ethics, and social groups such as ageing LGBTIQ+ (Poulsen et al., 2019; Poulsen, Burmeister, \& Tien, 2018).

Overall, the systematic review by Miah et al. (2019) provides invaluable insights into the current state of health informatics by using a systematic methodology and thematic analysis. Here, we have shared the reader and researcher perspective, identifying a few absent health 
informatics topics and complementing the findings by identifying them here using the authors' themes.

\section{Adam Poulsen}

Charles Sturt University

apoulsen@csu.edu.au

\section{Anwaar Ulhaq}

Charles Sturt University

\section{Response to the Post Publication Review}

The prime author of the review study (Miah et al. 2019) entirely agree to the Blockchain based new technological prospects in improving healthcare information services. The heath informatics field will be enriched in particular for the solution design arena, what was suggested.

The Blockchain technology was invented by Satoshi Nakamoto in 2008, nearly a decade ago (Nakamoto, 2008). Since then, its applications have been widely introduced and discussed in many sectors however, it is remarkable in the financial sector where the security, privacy and authorization are the vital issues. Despite this, the enormous potential of the application of Blockchain is still under exploration and the realisation of its benefits to many organisations and individuals are still at its emergent stages.

Government as well as enterprises are showing their increasing interests in adopting Blockchain based technologies realising that this technology may provide them effective support and further provisions for digital transformation. The World Economic Forum (WEC, 2018) viewed that the Blockchain would make industrial revolutions due to the facts that this technology can provide data security, transparency, as well as cost savings as the economic advantages. The main characteristics of the Blockchain are: it works in a decentralised manner, for wider ranges of data and their transactions ensuring authorisations and compatibilities that could offer useful provisions for designing various healthcare information dissemination solutions. Consequently, many recent studies discussed solution design using the Blockchain based applications in healthcare such as for health record management (Kar, 2016) and how Blockchains and smart contracts operate for identifying the advantages as well as highlighting the ways the Blockchains and other existing technologies such as IoT can be used together (Christidis and Devetsikiotis 2016). For improving user access management and data quality and providing access to patients for managing their own data offer options to integrate the informed consent procedure while ensuring privacy of data transactions (Razak, 2018). Along these lines, we anticipate that Blockchain based technology will make shift to transform the ways governments, businesses and communities use IT or Internet enabled platform as infrastructure for information collection, processing and analyzing that ensue effective delivery of information for better strategic decision support services. To capture the future research directions in the solution design research adopting Blockchain, we offer the following future aspects:

- $\quad$ Strategic decision making services: Further effective and robust decision support mechanisms development would be important study for the field of health informatics, in which structured and unstructured data are to be processed such as for setting out organisational vision and strategy as well as healthcare management to become more data-driven and vibrant solution applications ( for example 
through the adoption of various specialised DSS applications (e.g. in knowledge based DSS (Miah, 2008; Miah, Kerr, Gammack and Cowan, 2008)).

- Information disseminations services: Further effective Blockchain health informatics solutions oriented studies will be important for providing information distribution services to open up various development opportunities for individuals and organisations using user-centric and data-driven strategies (examples can be viewed in agricultural information services (Miah, 2012) and e-government (Miah, 2010)).

- Analytics support services: Further effective Blockchain health informatics solutions oriented studies will be important for developing data surveillance, business intelligence/analytics as well as predictive analytics for improving processes and practices as well as for better use of resources.

Let me conclude this brief commentary with a claim that for such solution design, studies may be benefited from a robust, broader and practice-focused design methodology in place. The design science research (DSR) paradigm (e.g. Miah and Gammack, 2014; Miah and Genemo 2016) provides better support in designing and evaluating specialised solutions over the past decades therefore, it would be showing promises for designing Blockchain based healthcare solutions. This pragmatism is a school of thought that considers practical consequences or realworld effects to be vital components of both practical meaning and truth (Hevner, 2007). Anderson (2013), cited in Tagle's e-book (2019, p.8) suggested that the pragmatic paradigm for design and development research is focused on practice which better aligns with the DSR. Based on these, it is anticipated that DSR would be a suitable methodological avenue for conducting Blockchain based healthcare solution design studies.

\section{Shah J Miah}

Victoria University, Melbourne

shah.miah@vu.edu.au

\section{References}

Anderson, M., Anderson, S. L., \& Berenz, V. (2017). A Value Driven Agent: Instantiation of a CaseSupported Principle-Based Behavior Paradigm. Paper presented at the AAAI 2016 Workshop on AI, Ethics \& Society.

Barik, R. K., Dubey, H., \& Mankodiya, K. (2017, 14-16 Nov. 2017). SOA-FOG: Secure serviceoriented edge computing architecture for smart health big data analytics. Paper presented at the 2017 IEEE Global Conference on Signal and Information Processing (GlobalSIP).

Burmeister, O. K., Islam, M. Z., Dayhew, M., \& Crichton, M. (2015). Enhancing client welfare through better communication of private mental health data between rural service providers. Australasian Journal of Information Systems, 19, 1-14. doi:10.3127/ajis.v19i0.1206

Carlson, B. L., Farrelly, T., Frazer, R., \& Borthwick, F. (2015). Mediating Tragedy: Facebook, Aboriginal Peoples and Suicide. Australasian Journal of Information Systems, 19. doi:10.3127/ajis.v19i0.1174

Christidis, K. \& Devetsikiotis, M. (2016). Blockchains and Smart Contracts for the Internet of Things, IEEE Access, 4, 2169-3536 
Gordon, W. J., \& Catalini, C. (2018). Blockchain Technology for Healthcare: Facilitating the Transition to Patient-Driven Interoperability. Computational and Structural Biotechnology Journal, 16, 224-230. doi:10.1016/j.csbj.2018.06.003

Hevner, A. R. (2007). A Three Cycle View of Design Science Research, Scandinavian Journal of Information Systems, 19(2), 87-92.

Kar, I. (2016). Estonian Citizens Will Soon Have the World's Most Hack-Proof Health-Care Records. [Online]. Available: http://qz.com/628889/this-eastern-european-country-ismoving-its-health-recordsto-the-blockchain/

Kraemer, F. A., Braten, A. E., Tamkittikhun, N., \& Palma, D. (2017). Fog Computing in Healthcare-A Review and Discussion. IEEE Access, 5, 9206-9222. doi:10.1109/ACCESS.2017.2704100

Kuo, T.-T., Kim, H.-E., \& Ohno-Machado, L. (2017). Blockchain distributed ledger technologies for biomedical and health care applications. Journal of the American Medical Informatics Association, 24(6), 1211-1220. doi:10.1093/jamia/ocx068

Miah, S. J., Shen, J., Lamp, J., Kerr, D., \& Gammack, J. (2019). Emerging Insights of Health Informatics Research: A Literature Analysis for Outlining New Themes. Australasian Journal of Information Systems, 23. doi:10.3127/ajis.v23i0.2137

Miah, S.J., \& Gammack, J. (2014). Ensemble Artifact Design for Context Sensitive Decision Support, Australasian Journal of Information Systems, 18 (2), 5-20

Miah, S.J., \& Genemo, H. (2016). A Design Science Research Methodology for Expert Systems Development, Australasian Journal of Information Systems, 20, 1-29

Miah, S.J. (2012). The role of end user in e-government application development: A conceptual model in the agricultural context, Journal of Organizational and End User Computing, 24 (3), 69-85

Miah, SJ (2010). A new semantic knowledge sharing approach for e-government systems, in the proceedings of the 4th IEEE International Conference on Digital Ecosystems and Technologies,

Miah, SJ, Kerr, D., Gammack, J. and Cowan, T. (2008). A generic design environment for the rural industry knowledge acquisition, Knowledge-Based Systems, 21 (8), 892-899

Miah, SJ (2008). An ontology based design environment for rural decision support, Unpublished PhD Thesis, Griffith Business School, Griffith University, Brisbane, Australia

Miah, S. J., Hasan, N., \& Gammack, J. (2017). Extending the framework for mobile health information systems Research: A content analysis. Information Systems, 69, 1-24. doi:10.1016/j.is.2017.04.001

Miah, S. J., Hasan, N., Hasan, R., \& Gammack, J. (2017). Healthcare support for underserved communities using a mobile social media platform. Information Systems, 66, 1-12. doi:10.1016/j.is.2017.01.001

Nakamoto, S. (2008). Bitcoin: A peer-to-peer electronic cash system. Consulted, 1: 2012

Poulsen, A. (2018). A Post Publication Review of "Threats to Autonomy from Emerging ICTs". Australasian Journal of Information Systems, 22. doi:10.3127/ajis.v22i0.1744 
Poulsen, A., Allen, M., Burmeister, O. K., Ul-Haq, A., Rehman, S., \& Tien, D. (2019). The role of care robots in managing loneliness among LGBT elderly. The Australian Nursing and Midwifery Journal.

Poulsen, A., \& Burmeister, O. K. (2019). Overcoming carer shortages with care robots: Dynamic value trade-offs in run-time. Australasian Journal of Information Systems, 23.

Poulsen, A., Burmeister, O. K., \& Tien, D. (2018). A new design approach and framework for elderly care robots. Paper presented at the Australasian Conference on Information Systems, Sydney, Australia.

Razak, O. (2018). Revolutionizing pharma - One blockchain use case at a time. Medium Corporation, URL: https://medium.com/linum-labs/revolutionizing-pharma-oneblockchain-use-case-at-a-time-e7922c8aa9a3

Simpson, C., Nevile, L., \& Burmeister, O. K. (2003). Doing Ethics: A Universal Technique in an Accessibility Context. Australasian Journal of Information Systems, 10(2). doi:10.3127/ajis.v10i2.159

Tagle, B. (2019). A Design Science Research Methodology Guide for graduate students, e-book published in URL: https://books.google.com.au/books?isbn=0359611729, accessed on 6 June 2019

Vanderelst, D., \& Winfield, A. (2018). An architecture for ethical robots inspired by the simulation theory of cognition. Cognitive Systems Research, 48, 56-66. doi:10.1016/j.cogsys.2017.04.002

WEC (2018). Building Block(chain)s for a Better Planet. In: ENVIRONMENT, P. A. S. W. I. F. T. (ed.) Fourth Industrial Revolution for the Earth Series. WEF.

Copyright: $@ 2019$ Poulsen, Ulhaq \& Miah. This is an open-access article distributed under the terms of the Creative Commons Attribution-NonCommercial 3.0 Australia License, which permits non-commercial use, distribution, and reproduction in any medium, provided the original author and AJIS are credited.

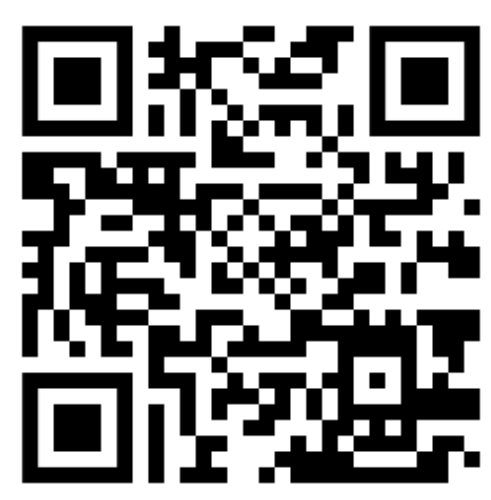

